\title{
A Capacitive Pressure Sensor Interface Using Oversampling $\Delta-\Sigma$ Demodulation Techniques
}

\author{
Mitsuhiro Yamada, Member, IEEE, and Kenzo Watanabe, Fellow, IEEE
}

\begin{abstract}
An interface for a capacitive pressure sensor which accommodates digital encoding and linearization functions has been developed. The front-end is a switched-capacitor bridge which converts the sensor capacitance into a proportional voltage. This voltage is applied to a pulse-width modulator to be compared with a model signal generated by a digital waveform synthesizer (DWS). This model-based signal processing provides the digital equivalent of the pressure being measured. For highyield, monolithic implementation of the interface, oversampling $\Delta-\Sigma$ demodulation techniques are used for the DWS. In addition to the digital functions, offset and scale adjustment, and crossparameter compensation are also possible with this architecture. A prototype interface is described to demonstrate these capabilities.
\end{abstract}

Index Terms - Capacitance measurement, capacitance transducer, digital filters, pressure measurement, pulse width modulation, sigma-delta demodulation, signal processing.

\section{INTRODUCTION}

B ECAUSE of the wide applicability in industrial processes, automobiles, and biomedical instrumentation, pressure sensors occupy a large share of the sensor market. To address this market, low-cost, mass-producible pressure sensors have been developed [1]. A typical sensor is the piezoresistor diffused onto a Si diaphragm. This type of integrated pressure sensor has played a major role in the early stage of transducer development because of its linear response and easy interfacing [2], [3]. Compared with the piezoresistive type, the capacitive pressure sensor, which detects a pressure by the capacitance change of a chamber, has such advantages as lower power dissipation, smaller temperature dependence, and higher sensitivity [4]-[6]. The capacitance change of the sensor is small, however, compared with the offset capacitance and is highly nonlinear. This presents several problems for the interface circuitry.

The first of these is the development of a front-end architecture which permits high-sensitivity detection of small capacitance changes. This problem has been solved by switchedcapacitor and oversampling analog-to-digital (A/D) conversion techniques [7], [8]. The second is the inverse problem of obtaining the pressure being measured from the detected sensor capacitance. As solutions to this problem, table look-

\footnotetext{
Manuscript received June 5, 1995; revised January 12, 1996. This work was supported by SMC Corp., Tsukuba, Japan.

M. Yamada is with the Department of Electrical and Electronic Engineering, Ibaraki University, Hitachi 316, Japan.

K. Watanabe is with the Research Institute of Electronics, Shizuoka University, Hamamatsu 432, Japan.

Publisher Item Identifier S 0018-9456(97)00915-7.
}

up and nonlinear encoding methods have been proposed [9]. These methods are valid for a one-dimensional (1-D) inverse problem, but their application to sensors which have a large temperature dependence is difficult. A promising approach to such a two-dimensional (2-D) or multidimensional inverse problem is model-based identification.

A capacitive pressure sensor can be modeled well by a simple expression [9], [10], and this expression can be easily transformed into the time domain by digital waveformsynthesis (DWS) techniques. Therefore, the model-based approach combined with switched-capacitor techniques seems the best solution to the interface problem for a capacitive pressure sensor. The accuracy in this approach depends solely on how precisely the model signal simulates the practical performance of the sensor. This usually requires high-precision components in the hardware realization. To reduce component precision requirements, and thereby to enable high-yield, monolithic implementation of the interface, oversampling demodulation techniques are used for generating the model signal. In the following sections, the capacitive pressure sensor interface designed based on these techniques is described.

\section{SENSOR MODEL}

A capacitive pressure sensor detects the applied pressure by means of the elastic deflection of the diaphragm. For the simple structure, the deflection is proportional to the applied pressure $P$. Therefore, the sensor capacitance $C(P)$ changes hyperbolically and is approximated well by

$$
\begin{aligned}
C(P) & =C_{0}+\Delta C(P) \\
& =C_{0}+C_{0} \frac{1-\alpha}{1-\frac{P}{P_{m}}} \frac{P}{P_{m}}
\end{aligned}
$$

where $C_{0}$ is the sensor capacitance when $P=0$, which is referred to as the offset capacitance hereafter; $\alpha$ is the sensitivity parameter depending on the detailed diaphragm structure; and $P_{m}$ is the maximum applicable pressure [9].

The sensor capacitance also changes with temperature. To a first-order approximation, the change due to temperature is linear and independent of the applied pressure. The sensor model for signal processing can thus be represented as

$$
\begin{aligned}
C(P, T) & =C_{0}(T)+\Delta C(P, T) \\
& =C_{0} f_{1}(T)+\Delta C\left(P, T_{0}\right) f_{2}(T)
\end{aligned}
$$

where

$$
f_{1}(T)=1+\beta_{1}\left(T-T_{0}\right)
$$




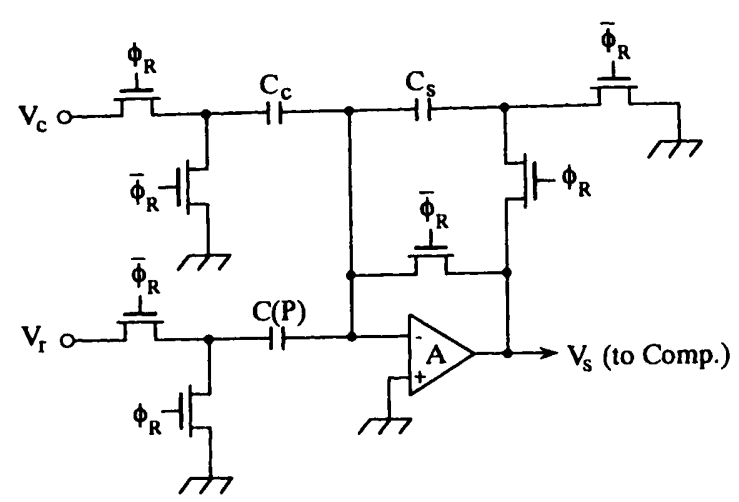

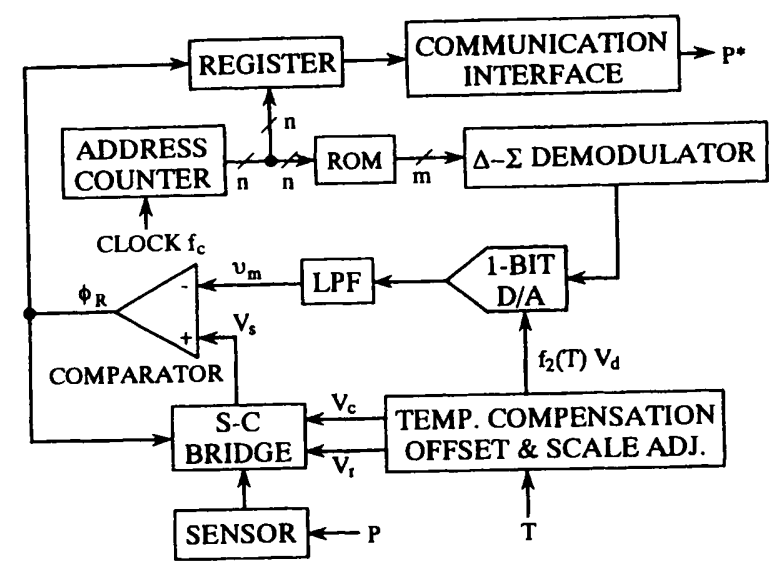

Fig. 1. A block diagram of the interface.

$$
f_{2}(T)=1+\beta_{2}\left(T-T_{0}\right)
$$

and $\Delta C\left(P, T_{0}\right)$ denotes the capacitance change due to the applied pressure at the reference temperature $T_{0}$, which is given by (1). It is noted that the temperature coefficients $\beta_{1}$ and $\beta_{2}$ assume different values in general.

\section{INTERFACE ARCHITECTURE}

A block diagram of the interface design based on the sensor model is shown in Fig. 1. The front-end is a switched-capacitor bridge. Canceling the offset capacitance $C_{0}(T)$, it converts the capacitance change $\Delta C(P, T)$ into a proportional voltage $V_{s}$. The incremental address counter, read-only memory (ROM), delta-sigma $(\Delta-\Sigma)$ demodulator, 1-bit digital-to-analog (D/A) converter, and low-pass filter (LPF) form the digital waveform synthesizer (DWS). The ROM stores the normalized sensor model $\Delta C\left(P, T_{0}\right) / C_{0}$. Transforming this model into the time domain, the DWS generates the model signal $v_{m}$. The comparator operates as the pulse-width modulator (PWM). The carrier signal to be modulated is the model signal $v_{m}$, and the modulation signal is $V_{s}$. Comparing $v_{m}$ and $V_{s}$, the comparator latches the digital equivalent of the pressure being measured into the register and sends it to an external device via the communication interface. Each block is described in more detail in the following sections.

Fig. 2 shows the circuit diagram of the switched-capacitor bridge. Here, $C(P)$ denotes the capacitive pressure sensor. Bridge operation is controlled by the reset signal $\phi_{R}$ issued by the comparator in Fig. 1. When $\bar{\phi}_{R}=$ " $1, " C(P)$ is charged to a reference voltage $V_{r}$ while $C_{c}$ and $C_{s}$ are discharged. The charge $C(P) V_{r}$ stored in $C(P)$ is then transferred to $C_{s}$ when $\phi_{R}=$ "1." At the same time, the charge $C_{c} V_{c}$ charges $C_{s}$ in the opposite direction. This process of charge transfer is insensitive to parasitic capacitances and the offset voltage of op-amp $A$, producing the output voltage

$$
V_{s}=\frac{C(P, T) V_{r}-C_{c} V_{c}}{C_{s}} .
$$

If the voltage $V_{c}$ is adjusted such that $C_{c} V_{c}=C_{0}(T) V_{r}$, then the offset capacitance $C_{0}(T)$ of the sensor is canceled. Temperature compensation is accomplished by introducing the temperature dependence $f_{1}(T)$ into $V_{c}$. The bridge thus
Fig. 2. The circuit diagram of the switched-capacitor bridge.

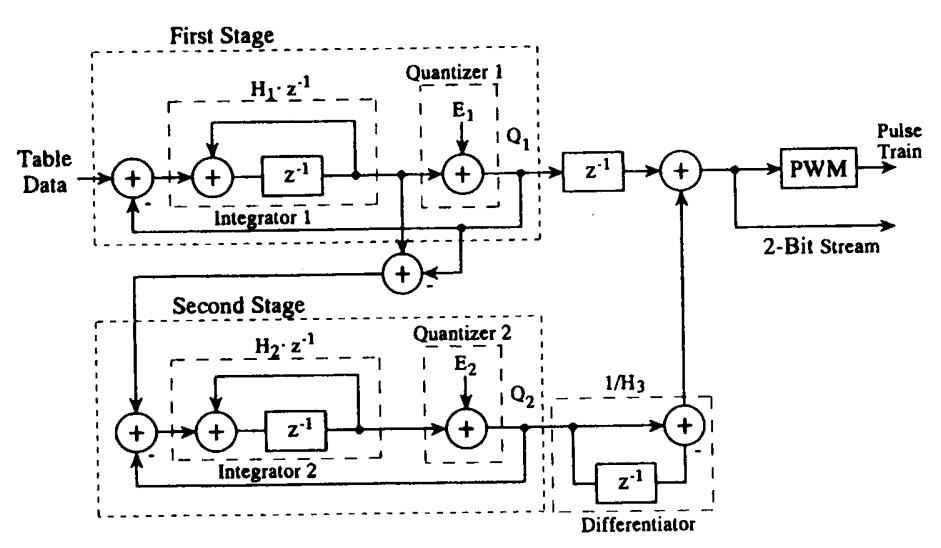

Fig. 3. The configuration of the oversampling $\Delta-\Sigma$ demodulator.

provides only the unbalance voltage due to the applied pressure

$$
V_{s}=\frac{\Delta C\left(P, T_{0}\right)}{C_{s}} f_{2}(T) V_{r}
$$

The ROM stores the sensor model in a one-dimensional table form. The address is the pressure $P^{*}$ quantized into sufficiently small steps, and the data is the normalized capacitance change $\Delta C\left(P^{*}, T_{0}\right) / C_{0}$ at the reference temperature $T_{0}$. The address size $n$ is determined by the required resolution $\varepsilon$, and if $\varepsilon=0.1 \%$, then $n=10$ bits. The data length depends not only on the required resolution but also on the nonlinearity of the sensor model. The nonlinearity can be measured in terms of the sensitivity of $\Delta C\left(P, T_{0}\right)$ to pressure $P$, which is defined by

$$
S_{p}^{\Delta C\left(P, T_{0}\right)}=\frac{\partial \ln \Delta C\left(P, T_{0}\right)}{\partial \ln P} .
$$

The nonlinearity index $k$ is given by the ratio of the maximum to minimum values of the sensitivity in the specified pressure range. If $k=1$, then the sensor is linear, and the required data length equals the address size. For $k$ other than 1, a data length of $\left(n+\log _{2} k\right)$ - or $\log _{2}(k / \varepsilon)$-bits is required to achieve the resolution $\varepsilon \%$. To our knowledge, the largest nonlinearity index of commercial capacitive pressure sensors is 4 .

The oversampling $\Delta-\Sigma$ demodulator follows the two-stage MASH configuration shown in Fig. 3 [11]. Containing two 1bit quantizers, it converts the table data in parallel form into the 2 -bit stream, assuming the four values, $-3,-1,+1$, and +3 . The 2-bit stream is then converted into the width-modulated pulse train according to the rule shown in Fig. 4 . The pulse train is finally converted into the analog model signal $v_{m}$ by the 1-bit D/A converter. For this demodulation technique to 


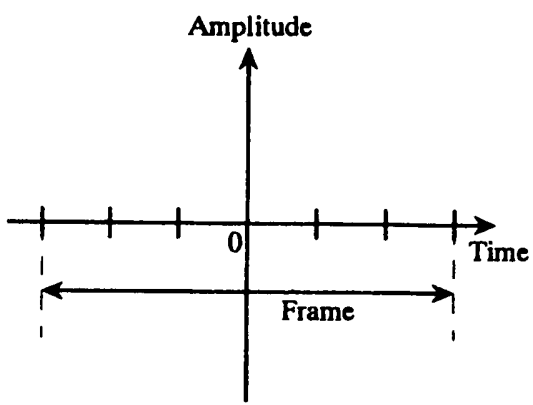

(a)-3

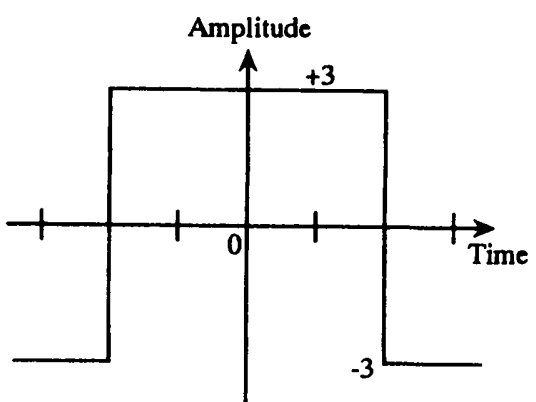

(c) 1

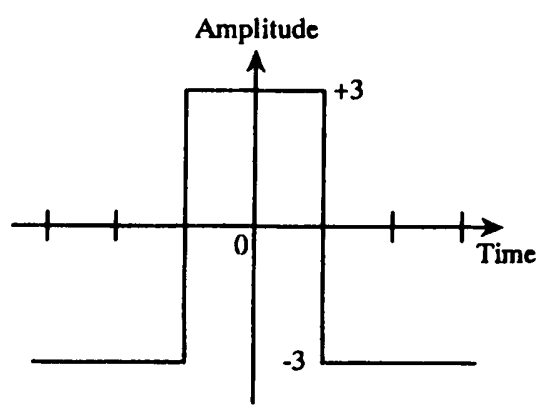

(b)-1

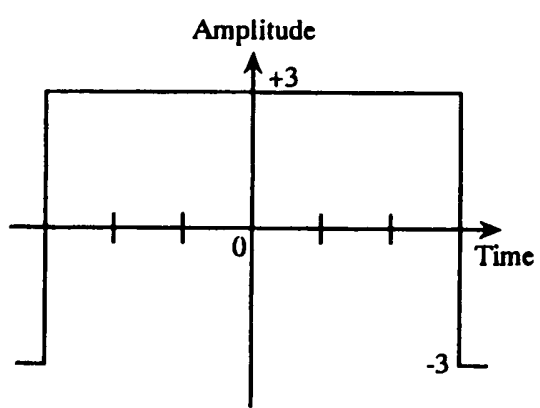

(d) 3

Fig. 4. The pulse-width representation of the oversampled 2-bit data.

be equivalent to that realized using a multibit D/A converter with $\varepsilon \%$ resolution, the quantization noise of the 1-bit D/A converter should be reduced more than $6.02 \log _{2}(k / \varepsilon) \mathrm{dB}$ by oversampling. To be specific, a signal-to-noise ratio (SNR) higher than $86.2 \mathrm{~dB}$ is required for the demodulator to achieve $0.1 \%$ resolution. The two-stage MASH demodulator improves the SNR by $15 \mathrm{~dB}$ by every doubling of the oversampling ratio (OSR). Therefore, the OSR should be higher than 100 to meet the resolution required for most applications. This limits the frequency spectrum of the model signal. The table data is read out of the ROM every clock cycle applied to the incremental address counter. The sampling frequency is thus the clock frequency $f_{c}$, and the Nyquist frequency is $f_{c} / 2$. To meet the SNR requirement, therefore, the model signal should be band-limited to $f_{c} / 2$ (OSR). In the DWS shown in Fig. 1, the band-limiting is accomplished by extrapolating the sensor model so that the table data results in a smooth, periodic function. This extrapolation technique will be described in the next section.

The LPF rejects the out-of-band noise. The third-order Bessel filter with a linear phase characteristic is used for exact waveform generation. The output is thus given by

$$
v_{m}(t)=\frac{\Delta C\left[P^{*}(t), T_{0}\right]}{C_{0}} f_{2}(T) V_{d}
$$

where the first term in the right-hand side is the sensor model stored in the address $P^{*}$, and $f_{2}(T) V_{d}$ is the reference voltage of the 1-bit D/A converter which introduces the temperature dependence $f_{2}(T)$. To indicate explicitly that $v_{m}$ is the sampled analog signal in synchronism with the clock frequency, time $t$ is included in (8). The scale adjustment is made by means of $V_{d}$. The comparator stores the address $P^{*}$ when $v_{m}$ coincides with $V_{s}$ into the register as the estimate of the pressure $P$ under measurement.

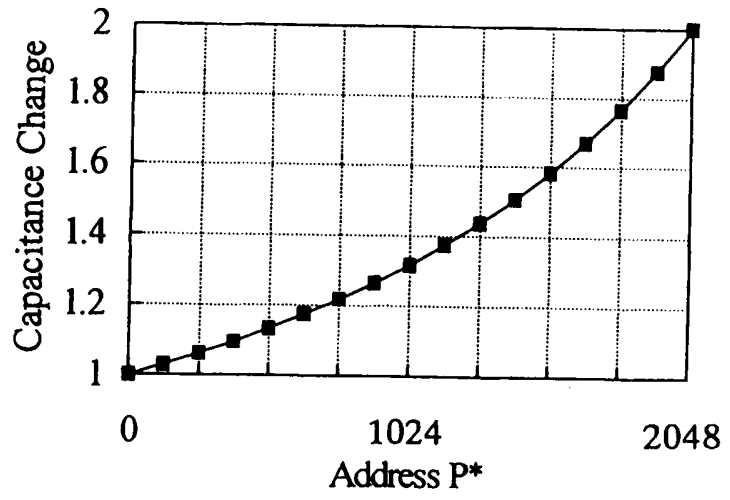

(a)

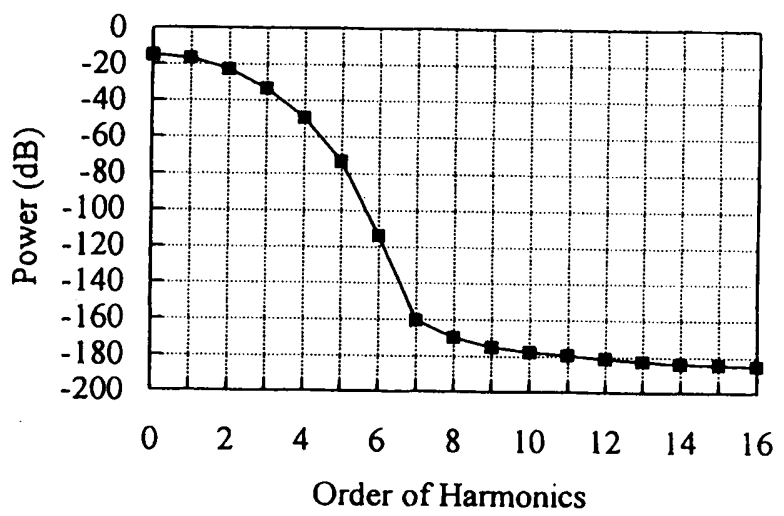

(b)

Fig. 5. The normalized capacitance change of (a) the pressure sensor and (b) its power spectrum.

\section{PROTOTYPE INTERFACE}

A prototype interface was built using off-the-shelf components. For easy assembly, the DWS was realized by using a $\mathrm{Z}-80 \mathrm{CPU}$. The resolution specified in the pressure range from 


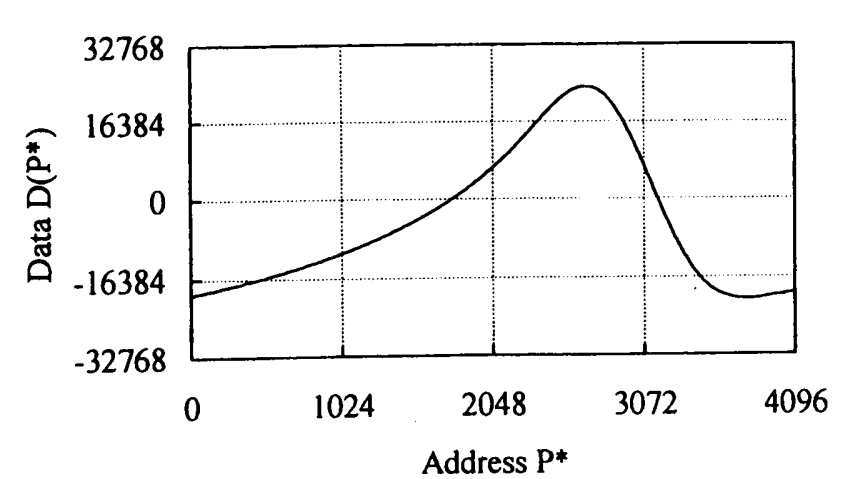

(a)

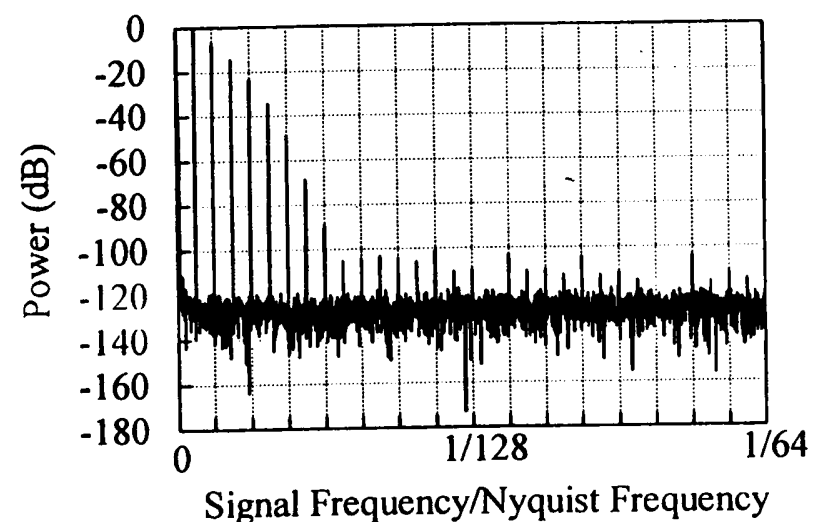

(b)

Fig. 6. The sensor model for (a) the oversampling demodulation and (b) its power spectrum.

$0-0.2 \mathrm{MPa}\left(2 \mathrm{~kg} / \mathrm{cm}^{2}\right)(\mathrm{FS})$ is $1 \% \mathrm{FS} .^{1}$ The model parameters of the pressure sensor were determined by measuring the pressure versus capacitance characteristics in the temperature range from $0-50^{\circ} \mathrm{C}: C_{0}=35.4 \mathrm{pF}, \alpha=0.443, P_{m}=3.81 \mathrm{kgf} / \mathrm{cm}^{2}$ $(0.374 \mathrm{MPa}), \beta_{1}=-1.2 \times 10^{-4}, \beta_{2}=1.5 \times 10^{-4}$. The temperature variations in the specified temperature range are within $0.5 \%$ FS. Therefore, no temperature compensation was made, although the interface accommodates such a function.

The sensor model $\Delta C\left(P, T_{0}\right) / C_{0}$ was calculated using these parameters. Fig. 5(a) shows the model that is stored in the ROM. For easy correspondence between the ROM address and pressure, the addresses from 0-2048 were allotted to the model. For the exact transformation of the model into the time domain, the power spectrum was calculated using a 2048-point FFT. A Kaiser window was applied to avoid spectrum broadening due to abrupt changes at the lower and upper bounds. The result is shown in Fig. 5(b). It indicates that the error accompanying the transformation will be of the order of $10^{-8}$ if the model signal includes the frequency components up to the 8th-order harmonic.

As explained in the previous section, the generation of such a model signal by means of an oversampling demodulator followed by the 1-bit D/A converter requires the model signal to be band-limited to $f_{c} / 2$ (OSR). To meet this requirement, the table size is expanded to 4096, and data at each address are determined as follows. First, data in the equally spaced 15 addresses in the expanded area are obtained by convolving the

\footnotetext{
${ }^{1}$ The international unit of pressure is $\mathrm{Pa}$. In this paper, however, $\mathrm{kgf} / \mathrm{cm}^{2}$ is used because of its easy correspondence with the table address.
}

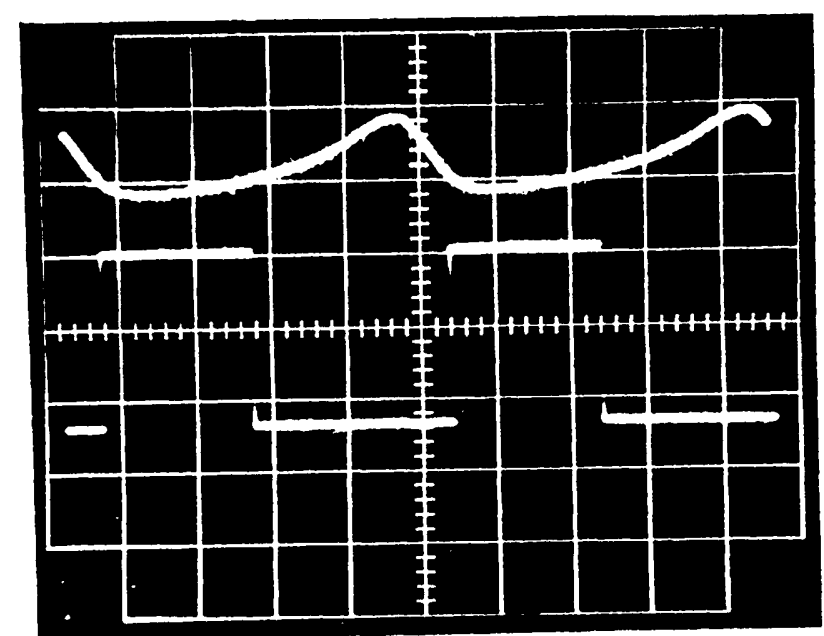

Fig. 7. Experimentally observed model signal (upper trace) and comparator output (lower trace). Vertical scale: $1 \mathrm{~V} /$ div (upper trace), $5 \mathrm{~V} /$ div (lower trace), and horizontal scale: $0.1 \mathrm{~s} / \mathrm{div}$.

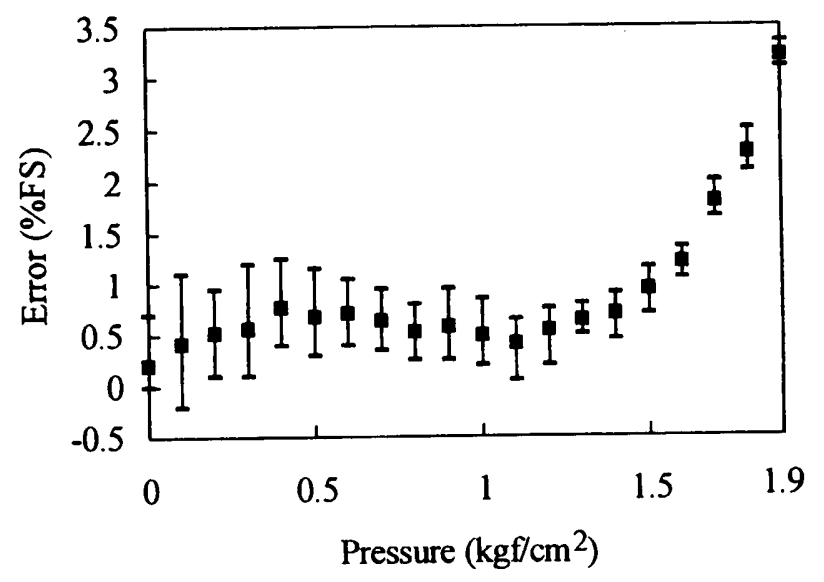

Fig. 8. Errors between readings of the prototype interface and a commercial manometer.

given 17 data points in Fig. 5(a) with the sampling function. As the sampling function, the impulse response of the lowpass FIR filter with 257 taps is used to prevent the convolution from generating the extra higher frequency components. The tentative data thus obtained are then optimized by the simplex method in linear programming [12], with the constraint

$$
\begin{aligned}
& \operatorname{minimize} \text { maximum }\left|D^{*}\left(P^{*}\right)-D\left(P^{*}\right)\right|, \\
& \quad\left(P^{*}=0,1,2, \cdots, 16\right)
\end{aligned}
$$

where $D\left(P^{*}\right)$ are data given in Fig. 5(a), and $D^{*}\left(P^{*}\right)$ represents data modified by the convolution. Data at the other addresses are finally determined by interpolation. The impulse response of the above-mentioned FIR filter is again used as the sampling function for the interpolation.

Fig. 6(a) shows the table data obtained by the above procedure. To enhance accuracy, 16 bits are used for the data length. Data at addresses from 0-2048 correspond exactly to the sensor characteristics shown in Fig. 5(a). The FFT analysis is shown in Fig. 6(b). The OSR for the 8th-order harmonic is 256 , which is enough for the SNR requirement. The clock frequency $f_{c}$ used for the DWS is $8.23 \mathrm{kHz}$. The Nyquist frequency in Fig. 6(b) is half the clock frequency, and the 8th-order harmonic frequency is $16 \mathrm{~Hz}$. The cutoff frequency 
of the Bessel filter is set to $20 \mathrm{~Hz}$. The sampling speed is $8.23 \mathrm{kHz} / 4096=2$ samples per second. Fig. 7 shows the model signal $v_{m}$ and the comparator output $\phi_{R}$ observed experimentally when $1 \mathrm{kgf} / \mathrm{cm}^{2}(0.98 \mathrm{MPa})$ pressure was applied to the sensor. The upper trace indicates that the DWS based on the above-described techniques generates the model signal successfully.

The digital equivalents of the applied pressure sent from the communication port of the Z-80 were displayed on 7-segment LED's and were compared with readings of a commercial manometer. The manometer is accurate to $0.5 \%$ FS. Errors between them are plotted in Fig. 8. It can be seen that errors are within $1 \%$ FS except at the higher pressure. The large error at the higher pressure is attributed to the deviation of the sensor model from the actual sensor characteristics. Errors are also offset by $0.5 \%$ FS. This offset is due to the incomplete cancellation of the offset capacitance. Including the higher order terms in the sensor model and the exact offset adjustment would improve the accuracy.

\section{CONCLUSIONS}

A capacitive pressure-sensor interface which provides the digital equivalent of the pressure to be measured has been presented. The provision of such a function, which would otherwise require the large number of precision components, was realized using a simple architecture. The key to this realization is model-based signal processing made possible by the DWS incorporating linear programming and oversampling $\Delta-\Sigma$ demodulation techniques.

The proposed architecture accommodates almost all the functions required for an interface and is applicable to any sensor provided that the appropriate model is stored in table form and that the front-end produces a sensor signal consistent with the model signal. Therefore, it is a promising candidate for a universal interface or a sensor signal processor. The resolution achievable with this architecture depends largely on a sensor model embedded in a ROM, and the best approach to an exact model may well be automatic tuning by learning.

\section{REFERENCES}

[1] S. Middlehoek and S. A. Audet, Silicon Sensors. London, U.K.: Academic, 1989, ch. 3 .

[2] K. Samaun, D. Wise, and J. B. Angell, "An IC piezoresistive pressure sensor for biomedical instrumentation," IEEE Trans. Biomed. Eng., vol. BMC-20, pp. 101-109, Mar. 1973.

[3] S. Sugiyama, M. Takigawa, and I. Igarashi, "Integrated piezoresistive pressure sensor with both voltage and frequency outputs," Sens. Actuators, vol. 4, pp. 113-120, 1983.
[4] Y. S. Lee and K. D. Wise, "A batch-fabricated silicon capacitive pressure sensor with low temperature sensitivity," IEEE Trans. Electron Devices, vol. ED-29, pp. 42-48, Jan. 1982.

[5] W. H. Ko, "Solid-state capacitive pressure transducers," Sens. Actuators, vol. 10 , pp. 303-320, 1986

[6] S. K. Clark and K. D. Wise, "Pressure sensitivity in anisotropically etched thin-diaphragm pressure sensors," IEEE Trans. Electron Devices, vol. ED-26, pp. 1887-1896, Dec. 1979.

[7] H. Matsumoto, H. Shimizu, and K. Watanabe, "A switched-capacitor charge balancing analog-to-digital converter and its application to capacitance measurement," IEEE Trans. Instrum. Meas., vol. IM-36, pp. 873-878, Dec. 1987.

[8] C. A. Leme, P. Malcovati, and H. Baltes, "Oversampled interface for IC sensors," in Proc. Instrumentation and Measurement Tech. Conf., 1994, pp. $652-655$.

[9] M. Yamada, T. Takebayashi, S. Notoyama, and K. Watanabe, "A switched-capacitor interface for capacitive pressure sensors," IEEE Trans. Instrum. Meas., vol. 41, pp. 81-86, Feb. 1992.

[10] J. C. Patra, G. Panda, and R. Baliarsingh, "Artificial neural networkbased nonlinearity estimation of pressure sensors," IEEE Trans. Instrum. Meas., vol. 43, pp. 874-881, Dec. 1994.

[11] K. Uchimura, T. Hayashi, T. Kimura, and A. Iwata, "Oversampling Ato-D and D-to-A converters with multistage noise shaping modulators," IEEE Trans. Acoust., Speech, Signal Processing, vol. 36, pp. 1899-1905, Dec. 1988.

[12] W. H. Press, B. P. Flannery, S. A. Teukolsky, and W. T. Vetterling, Numerical Recipes in C. Cambridge, U.K.: Cambridge Univ. Press, 1988, ch. 10.

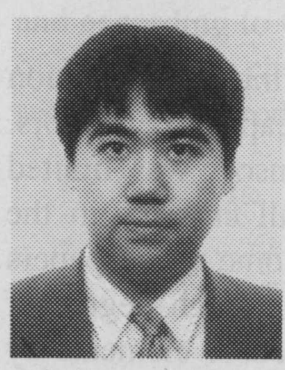

Mitsuhiro Yamada (M'95) received the B.E., M.E., and Dr.E. degrees from Shizuoka University, Hamamatsu, Japan, in 1990, 1992, and 1995, respectively.

He has been an Assistant, Department of Electrical and Electronic Engineering, Ibaraki University, Hitachi, Japan, since 1995. His research interests include sensor signal processing, evolvable hardware, and neural networks.

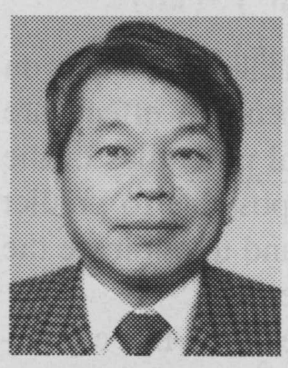

Kenzo Watanabe (M'74-SM'86-F'93) received the B.E. and M.E. degrees in engineering from Shizuoka University, Hamamatsu, Japan, in 1962 and 1966, respectively, and the Dr.Eng. degree from Kyoto University, Kyoto, Japan, in 1976.

Except for a year as a Visiting Professor at the University of California, Los Angeles, he has been on the faculty at Shizuoka University since 1962, serving progressively as Research Assistant, Associate Professor, and Professor. $\mathrm{He}$ is now a Professor, Research Institute of Electronics,

Shizuoka University.

Dr. Watanabe serves as an AdCom member of the Instrumentation and Measurement Society, an Associate Editor of the IEEE TRANSACTIONS ON INSTRUMENTATION AND MEASUREMENT, and a member of the IMTC Board of Directors. 